POS $\quad$ PROCEEDINGS

\title{
Cosmogenic Neutrinos
}

\section{Todor Stanev*}

Bartol Research Institute and Department of Physics and Astronomy, University of Delaware, Newark, DE 19716, U.S.A.

E-mail: stanev@bartol.udel.edu

We discuss the interactions of ultrahigh energy cosmic rays with photon fields in propagation to us from their sources and the secondary particles produced in such interactions. We identify the astrophysical parameters that the number of secondary particles depends on and demonstrate the fluxes that different models of the highest energy cosmic ray can produce.

$X V$ Workshop on Neutrino Telescopes,

11-15 March 2013

Venice, Italy

\footnotetext{
*Speaker.
} 


\section{Introduction}

There are two types of astrophysical neutrinos that we expect to observe in the future: neutrinos produced at the sources where cosmic rays are accelerated, and diffuse neutrinos. From galactic cosmic ray sources, such as supernova remnants, we expect to see neutrinos from the decay of charged pions and kaons produced in hadronic interactions of the accelerated cosmic rays with the matter surrounding the sources. $\mathrm{TeV}$ gamma rays that are generated by the neutral mesons decays have been seen from such objects.

Since we do not expect to have large amounts of matter in the vicinity of the extragalactic cosmic ray sources, such as Active Galactic Nuclei (AGN), the expectations are that cosmic rays accelerated there would interact with the intense radiation fields and produce $\gamma$-rays and neutrinos. Such interactions are called photoproduction. The threshold energy for photoproduction in the center of mass system of the interaction $\sqrt{s}$ should be at least equal to the sum of the proton and pion masses $m_{\pi}+m_{p}$, which is $1.08 \mathrm{GeV}$. The center of mass energy squared $s$ in photoproduction interactions is

$$
s=m_{p}^{2}+2 E_{p} \varepsilon\left(1-\beta_{p} \cos \theta\right),
$$

where $E_{p}$ is the proton energy in the Laboratory system, $\varepsilon$ is the energy of the photon, and $\theta$ is the angle between the two interacting particles. When the particles go exactly against each other $\cos \theta$ $=-1$ and this defines the lowest proton energy. Photoproduction interactions have been extensively studied in the 1960's and their cross section is well established to quite high CMS energy. The accelerator experiments were performed with $\gamma$-rays interacting on proton target in the so called Nucleon Rest Frame (NRF) system. The minimum photon energy for photoproduction in the NRF is $145 \mathrm{MeV}$.

For $\varepsilon=1 \mathrm{eV}$ (optical radiation) the minimum proton energy is slightly higher than $7 \times 10^{16}$ $\mathrm{eV}$, i.e. all extragalactic objects that are seen with optical telescopes and can accelerate protons to $10^{17} \mathrm{eV}$ would produce $\gamma$-rays and neutrinos. Waxman\&Bahcall [1] were the first authors to use photoproduction interactions and calculate the neutrino fluxes generated by the extragalactic cosmic ray sources. Using the average fraction of the proton energy that the generated neutrinos carry they obtained the maximum $v_{\mu}$ and $\bar{v}_{\mu}$ neutrino flux generated by extragalactic sources to be

$$
E_{v}^{2} \Phi_{v}=1.5 \times 10^{-8} \mathrm{GeV} \cdot \mathrm{cm}^{-2} \cdot \mathrm{srad}^{-1} \cdot \mathrm{s}^{-1}
$$

Including a $(1+z)^{3}$ cosmological evolution (where $z$ is the redshift) of the sources they obtained a limit of $E_{v}^{2} \Phi_{v}$ of $4.5 \times 10^{-8} \mathrm{GeV} /\left(\mathrm{cm}^{2}\right.$.srad.s $)$. Although we disagree with many assumptions in this calculation (including the assumption that all cosmic rays are accelerated on a $E^{-2}$ spectrum) this provides us with a useful straight line in $E_{v}^{2} P h i_{v}$ to compare to observations and more sophisticated models.

Ultrahigh Energy Cosmic Rays (UHECR) are the highest energy fraction of the cosmic rays that are accelerated (we assume) at extragalactic cosmic ray sources. They can have photoproduction interactions on the most universal Cosmic Microwave Background (CMB), the leftover from the beginning of the Universe, which currently has a temperature of $2.725^{\circ} \mathrm{K}$. This temperature corresponds to an average energy of the $\mathrm{CMB}$ of $6.34 \times 10^{-4} \mathrm{eV}$. Using the same simple calculation for the minimum proton energy for interactions in $\mathrm{CMB}$ we obtain $10^{20} \mathrm{eV}$. The actual minimum energy is lower $\left(3 \times 10^{19} \mathrm{eV}\right)$ as the CMB energy distribution extends in a blackbody spectrum above 
$10^{-3} \mathrm{eV}$. The existence of such neutrinos, usually called cosmogenic was suggested by Berezinsky\&Zatsepin in 1969 [2] and independently by Stecker [3].

Since the pioneering work of Berezinsky, Zatsepin \& Stecker they have been quite many calculations of the expected fluxes of cosmogenic neutrinos. Among the older ones the most interesting work is that of Ref. [4] that was done with the aim to use the neutrino flux to estimate the cosmological evolution of the cosmic ray sources. The current question is not if the cosmogenic neutrinos exist, we believe they do, but what is their flux. This depends on a large number of astrophysical parameters that we will define later.

\section{Neutrino production by UHECR on propagation}

The first step in all proton propagation calculations in the CMB is the calculation of the mean free path $\lambda$ of the protons as a function of their energy. It is given by

$$
\lambda_{p \gamma}^{-1}\left(E_{p}\right)=\frac{1}{8 E_{p}^{2}} \int_{\varepsilon_{t h r}}^{\infty} d \varepsilon \frac{n(\varepsilon)}{\varepsilon^{2}} \int_{s_{\min }}^{s_{\max }} d s\left(s-m_{p}^{2}\right) \sigma_{p \gamma}(s)
$$

where $\varepsilon$ is the photon energy in $\mathrm{eV}, n(\varepsilon)$ is the photon number density in $\mathrm{cm}^{-3} \mathrm{eV}^{-1}$. The mean free path has a minimum of $3.8 \mathrm{Mpc}$ at $\mathrm{E}_{p}$ about $5 \times 10^{20} \mathrm{eV}$ and slightly increases at higher energy. At relatively small $\sqrt{s}$ the main process that dominates the cross section is $p \gamma \rightarrow \Delta^{+}$which has a cross section over $500 \mu \mathrm{b}$. The resonance $\Delta^{+}$decays either to $p \pi^{0}$ or to $n \pi^{+}$with a ratio between the two decay channels of 2 . Then a 2 of heavier resonances follow with lower cross section and the multiparticle production starts at about $\sqrt{s}$ of about $5 \mathrm{GeV}$. It increases with the interaction energy and is roughly $1 \%$ of the $p-p$ cross section.

Another very important photoproduction interactions parameter is the proton inelasticity 0 $\mathrm{K}_{\text {inel }}$ which defines the average fraction of its energy which the proton loses in the interaction and which goes in the production of secondary particles. At low $\sqrt{s}$ this fraction is small. $\mathrm{K}_{\text {inel }}$ is 0.17 for $10^{20} \mathrm{eV}$ protons interacting in CMB. The distribution of this quantity is between 0 and 0.4 , i.e. there are no cases where the proton loses more than $40 \%$ of its energy. Above $10^{20} \mathrm{eV} \mathrm{K}_{\text {inel }}$ increases and at $10^{21} \mathrm{eV}$ it grows to 0.27 and the distribution becomes flatter reaching inelasticities of $60 \%$.

The mean free path $\lambda_{p \gamma}$ and $\mathrm{K}_{\text {inel }}$ define the mean loss length: the distance at which a proton loses all its energy in interactions on the photon background. It is

$$
L_{\text {loss }}=\frac{E_{p}}{d E_{p} / d x}=\frac{\lambda_{p \gamma}\left(E_{p}\right)}{K_{\text {inel }}\left(E_{p}\right)} .
$$

The minimum of $L_{\text {loss }}$ coincides with the position of the minimum $\lambda_{p \gamma}$ and at $E_{p}=5 \times 10^{20} \mathrm{eV}$ it is about $16 \mathrm{Mpc}$ and remains roughly constant as $\mathrm{K}_{\text {inel }}$ increases.

In calculating the $\gamma$-ray and neutrino fluxes from UHE protons propagating in CMB and other universal photon backgrounds one also has to account for the other processes in which these protons lose energy. The main such process is $p \gamma \rightarrow e^{+} e^{-}$. The energy loss length for this process is much larger, never below $100 \mathrm{Mpc}$. The mean free path is small but the energy loss in each interaction is tiny. In propagation on cosmological distances one also has to account for the proton adiabatic energy loss from the expansion of the Universe. 


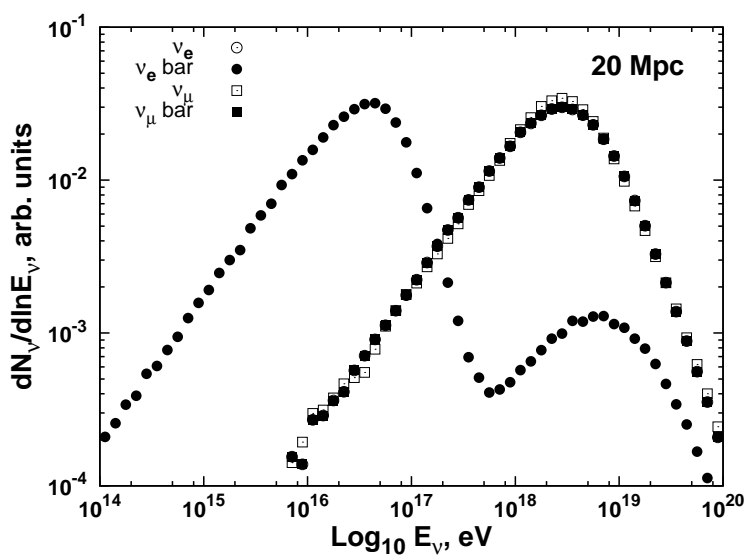

Figure 1: Spectra of different neutrino types generated in proton propagation on $20 \mathrm{Mpc}$ in CMB. The energy spectrum of the injected protons is $\mathrm{E}^{-2.5}$. See text for different symbols.

Figure 1 shows the spectra of neutrinos generated by an $\mathrm{E}^{-2.5}$ proton spectrum propagated at distance of $20 \mathrm{Mpc}$. Electron neutrinos are indicated with open circles, $\bar{v}_{e}$ - with filled circles, $v_{\mu}$ with open squares, and $\bar{v}_{\mu}$ with closed squares. The spectra of $v_{e}, v_{\mu}$ and $\bar{v}_{\mu}$ are almost identical, so close to each other that the $v_{e}$ points are almost invisible. The electron antineutrinos have a very different spectrum that 3 slightly above $10^{16} \mathrm{eV}$. The reason is that $\bar{v}_{e}$ are only generated in neutron decay $\left(n \rightarrow p e^{-} \bar{v}_{e}\right)$. The neutron decay length is smaller than the neutron interaction length up to energy of $4 \times 10^{20} \mathrm{eV}$. A small peak at about $5 \times 10^{18} \mathrm{eV}$ is created in neutron interactions in CMB.

\section{Energy spectra of cosmogenic neutrinos}

The best way of calculating the cosmogenic neutrinos energy spectra is to propagate protons on different distances and then integrate the results of the propagation using the astrophysical assumptions [5]. Figure 2 shows the result of the integration for a proton injection spectrum of $\mathrm{E}^{-2.5}$ and a modest cosmological evolution of $(1+z)^{2}$. The thick solid line shows the sum of all neutrino

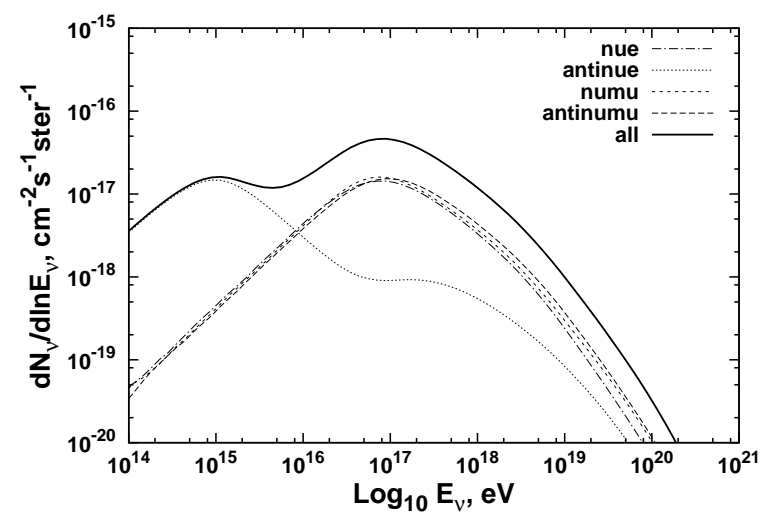

Figure 2: Spectra of cosmogenic neutrinos integrated on propagation on redshifts from 0 to 8 in the CMB. The proton injection spectrum extends to $10^{21.5} \mathrm{eV}$. 
types. It is interesting to understand what contributes the most to these energy spectra. The highest energy neutrinos are generated in the present Universe, close to $z=0$ because neutrinos generate at large redshifts have their energy scaled down by $(1+z)$. The flux of $\bar{v}_{e}$ increases and its maximum moves to higher energy as higher energy neutrons also decay. There is a strong dependence of these effects on the cosmological evolution of the sources [6] and a moderate dependence on the cosmological model.

Here is the list of all parameters one needs to perform a calculation.

- The total emissivity of the Universe in UHE cosmic rays, usually expressed in ergs/ $/ \mathrm{Mpc}^{3} / \mathrm{year}$. This a coefficient that normalizes the calculation to the assumed proton flux in the Universe.

- The average acceleration spectrum of these particles. The flatter the spectrum is the more UHECR can interact in the CMB.

- The chemical composition of UHECR. This relates to the spectrum of protons because nuclei interact in the CMB in a different way that we will briefly discuss later.

- The maximum acceleration energy in the UHECR sources. It is obvious that the higher the maximum energy is, there are more photoproduction interactions and more (and higher energy) neutrinos are generated.

- The cosmological evolution of the UHECR sources.

Many of these parameters are related. High maximum acceleration energy and a flat acceleration spectrum generate higher emissivity of UHECR, for example. A strong cosmological evolution can compensate for a low current emissivity at high redshifts when the energy of the CMB was higher and lower energy protons were able to photoproduce. It is difficult to describe all relations between these five parameters.

Figure 3 shows the spectra of muon neutrinos and antineutrinos generated on propagation in $200 \mathrm{Mpc}$ in the $\mathrm{CMB}$ as a function of the maximum acceleration energy of the protons. The proton injection spectrum is $\mathrm{E}_{p}^{-2.5}$. The difference between $\log \left(E_{p}^{\max }\right)$ of $10^{21.5}$ and $10^{20} \mathrm{eV}$ is not big because of the relatively flat injection spectrum. If the acceleration spectrum were flatter the difference would increase. Less and less neutrinos are generated when the maximum energy decreases and the neutrino spectrum also changes. It is impossible to produce neutrinos of energy higher than $\mathrm{E}_{p}^{\max }$, or course and for this reason the energy spectrum is narrower for the lowest maximum energy.

\subsection{The other universal photon background}

The cosmic microwave background is not the only universal photon background. There is also the extragalactic background light that covers to wavelengths between the microwave and optical radiation. It exhibits two peaks: one at the maximum of the optical light close to energy of one $\mathrm{eV}$, and another above wavelengths of $100 \mu \mathrm{m}$ that represents the scattered optical light. The total number density of EBL is about $1 \mathrm{~cm}^{-3}$, more than 400 times lower than that of the CMB, but even the far infrared has energy much higher than that of the CMB. That means that much lower energy protons would photoproduce in the EBL and also generate neutrinos. The energy spectra of 


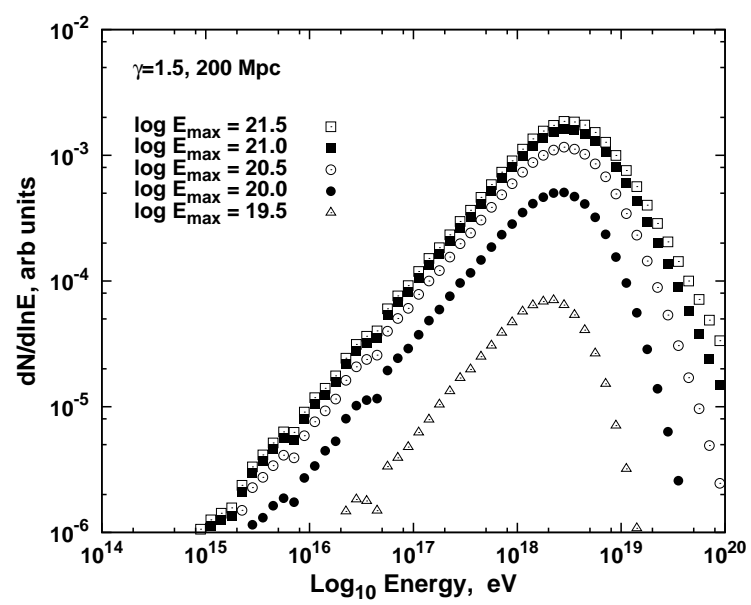

Figure 3: Spectra of cosmogenic $v_{\mu}$ and $\bar{v}_{\mu}$ produced on propagation on $200 \mathrm{Mpc}$ (redshift $z$ of 0.05 ) of proton spectra with different maximum energy.

the cosmogenic neutrinos generated in EBL will have roughly the same shape but will be shifted to lower energy than these generated in the CMB. While the lowest energy protons interacting in the CMB is $4 \times 10^{19} \mathrm{eV}$ even $10^{17} \mathrm{eV}$ protons occasionally interact in the EBL - see the estimates in Section 1 for photons of energy $1 \mathrm{eV}$.

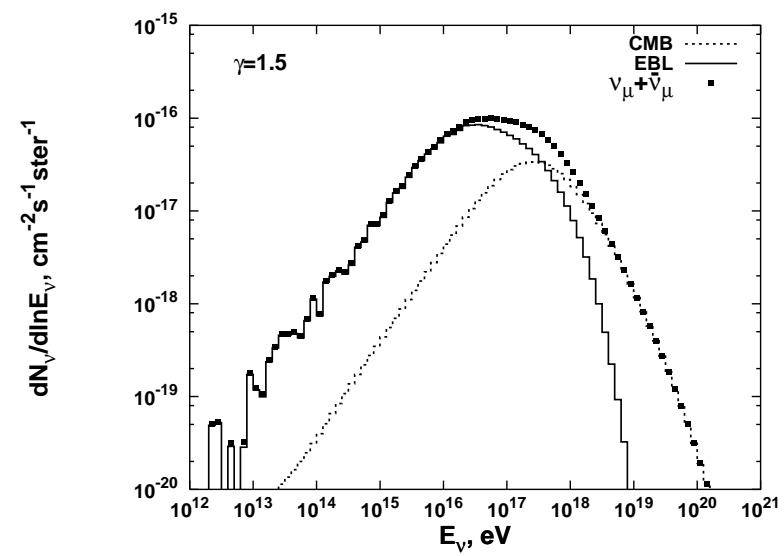

Figure 4: Spectra of cosmogenic $v_{\mu}$ and $\bar{v}_{\mu}$ produced on propagation in the CMB and in the EBL. The points show the sum of the neutrinos generated in the two targets.

The fact that lower energy protons interact in EBL has an interesting effect on the neutrino production: while in interactions on the $\mathrm{CMB}$ flatter injection spectra generate more neutrinos, in interactions in EBL steeper proton spectra have the same effect since there are more lower energy protons for the same emissivity. The steeper the proton spectrum is the bigger the contribution of the EBL target is.

\subsection{Interactions of nuclei in photon fields}

Nuclei heavier than protons have also another source of energy loss - photodisintegration. The dominant process is the giant dipole resonance induced in the nuclei by the microwave background 
or any other photon field. The giant dipole resonance cross-section peaks in the $\varepsilon^{\prime}$ energy range 10-30 MeV. The nucleus absorbs the photon and forms an excited state, which decays, releasing one or two nucleons. The photoabsorption cross-section roughly obeys the Thomas-Reiche-Kuhn sum rule. It is usually defined as

$$
\sigma_{\text {phabs }} \equiv \int_{0}^{\infty} \sigma\left(\varepsilon^{\prime}\right) d \varepsilon^{\prime} \simeq 60 \frac{N Z}{A}
$$

The photoabsorption cross-section $\sigma_{\text {phabs }}$ is measured in mb.MeV. In (3.1) $A$ is the mass number, $Z$ is the charge and $N$ is the number of neutrons.

This is only a rough approximation of the real cross-section that depends on the stability of the nucleus. At energies, $\varepsilon^{\prime}$, lower than about $30 \mathrm{MeV}$ the disintegration is dominated by the emission of one or two nucleons. At higher energy the emission of more than two nucleons is possible.

Generally, because of its charge, it appears easier to emit a proton than a neutron. Stable nuclei are more difficult to disintegrate, although there are no absolute rules. It is even more important to account to the $e^{+} e^{-}$pair production energy loss since it scales as the nucleus charge $Z^{2}$.

It is obvious that a nucleus of total energy $10^{20} \mathrm{eV}$ will have energy per nucleon $A$ times smaller and iron nuclei of that energy will never suffer photoproduction and generate high energy cosmogenic neutrinos. On the other hand, many of the neutrons released in the photodisintegration will decay and generate $\bar{v}_{e}$. For that reason the fluxes of electron antineutrinos increase significantly if the composition of the UHECR is heavy or mixed, while the fluxes of high energy neutrinos decrease.

\section{Expected fluxes of cosmogenic neutrinos}

The expected fluxes of cosmogenic neutrinos are very different depending on the interpretations on the cosmic ray energy spectrum and composition detected by the UHECR detectors, such as the Auger observatory [7], the High Resolution Fly's Eye [8] and the Telescope array [9]. While the energy estimates of Auger and the other two detectors are only different by about $20 \%$ (with difference decrease reported at the International Cosmic Ray Conference in 2013) they do measure different cosmic ray composition above $10^{18} \mathrm{eV}$. HiRes and TA measure a very light cosmic ray composition that would generate cosmogenic neutrinos. The Auger observatory claims a mixed composition above $10^{18} \mathrm{eV}$ that becomes increasingly heavier above $10^{19} \mathrm{eV}$. The statistics is insufficient to have a better model of the chemical composition as a function of the primary energy, but the tendency is to observe a larger fraction of heavy nuclei at the highest energies. Since this energy range coincides with threshold energy for neutrino production in the CMB (see Fig. 3, the expected flux of cosmogenic neutrinos is low.

In addition, the models for the generation of the UHECR energy spectrum also strongly influence the neutrino production. In classical models where the dip of the spectrum at about $3 \times 10^{18}$ $\mathrm{eV}$ is due to the emergence of extragalactic cosmic rays, the extragalactic injection spectrum would be about $\mathrm{E}^{-2.3}$ and may require a strong cosmological evolution of the sources. On the other hand, the dip model of Ref. [10], which explains a proton dominated spectrum that fits the observations down to $10^{17} \mathrm{eV}$, uses injection spectrum with index -2.7 and requires no cosmological evolution of the sources. 
Figure 5 compares the prediction of the two extreme interpretations of the energy spectrum: the fit of the Auger spectrum [11] with protons accelerated to a -2.3 spectral index with $(1+z)^{5}$ cosmological evolution and that of Ref. [10] with $\mathrm{E}^{-2.7}$ acceleration and no cosmological evolution. The latter model fits well the spectrum measured by the HiRes experiment [12]. The UHECR emissivity above $10^{19} \mathrm{eV}$ used in the calculation in both models is $1 / 2$ of that defined in [1].

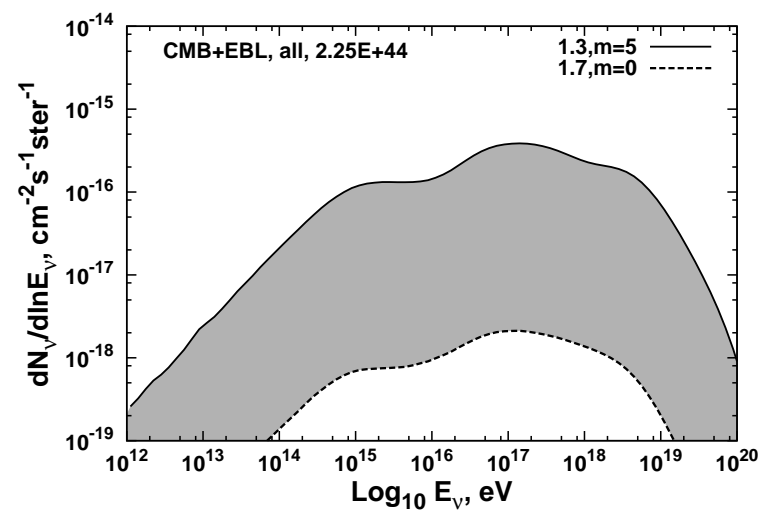

Figure 5: Spectra of all cosmogenic neutrinos generated by the two extreme models described in the text. Both models have purely proton chemical composition. All interactions on the CMB and in the EBL are included in the neutrino production.

One can easily see that the difference in the neutrino flux is more that two orders of magnitude in almost the whole energy range. It is true that the maximum $(\gamma=1.3, m=5)$ model is not very realistic. We show it here to emphasize the huge difference in the expectations.

A very good discussion of the dependence of the cosmological neutrino flux on the UHECR composition is made in Ref. [13], which describes the disappointing model in which the highest energy cosmic rays are iron nuclei. In such a model, its authors say, the maximum energy of the accelerated protons is between two and five $\mathrm{EeV}(2-5) \times 10^{18} \mathrm{eV}$ and the highest energy iron nuclei are $2.8 \times 10^{20} \mathrm{eV}$. Therefore no neutrinos would be produced in current interactions with the CMB photons. There would still be some production at high redshifts, in the EBL and, of course, $\bar{v}_{e}$ from neutron decay. If the disappointing model is true, there is no chance that IceCube [14] will ever detect cosmogenic neutrinos.

The interest in detecting cosmogenic neutrinos rose substantially last year when IceCube announced the detection of two $10^{15} \mathrm{eV}$ neutrino induced cascades [15]. Since this energy is significantly lower than $10^{18} \mathrm{eV}$ the main question was if these two events are not $\bar{v}_{e}$ interacting with electrons to generate the Glashow Resonance $\bar{v}_{e}+e^{-} \rightarrow W^{-}$. The resonant cross section reaches $0.47 \mu \mathrm{b}$ at $6.4 \times 10^{15} \mathrm{eV}$ but the width of the resonance is very narrow $2.1 \mathrm{GeV}$. The $W^{-}$decays in six hadronic and three leptonic channels, but all of them would create significantly higher energy cascades in IceCube. Eventually the conclusion was that these are not likely to be $\bar{v}_{e} e$ interactions.

Limits of the cosmogenic neutrinos of energy above $10^{17} \mathrm{eV}$ have been set by the Auger air shower array [16], the RICE experiment at the South Pole [17] and by the ANITA experiment [18]. These limits are significantly higher than the most cosmogenic neutrino models. Auger has updated their neutrino limit on the basis of six years observations by several different air shower techniques [19]. The new differential limit for a single neutrino flavor is $8 \times 10^{-17}$ in the same units 
as Fig. 5. To compare with the predictions for all neutrino species shown in this figure it seems to touch exclude the highest predicted neutrino flux.

A possible future detection of cosmogenic neutrinos would not only benefit neutrino physics. It will contribute to many general astrophysical communities dealing with cosmic ray acceleration, possible sources of UHECR, and propagation in the Universe.

Acknowledgments This work is funded in part by the US Department of Energy grant DE-FG0291ER40626.

\section{References}

[1] E. Waxman \& J.N. Bahcall, Phys. Rev. D59:023002 (1999)

[2] V.S. Berezinsky \& G.T. Zatsepin, Phys. Lett. 28B, 423 (1969)

[3] F.W. Stecker, Astrophys. Sp. Sci., 20, 47 (1973)

[4] C.T. Hill \& D.N. Schramm, Phys. Lett. B 131, 247 (1983)

[5] R. Engel, D. Seckel \& T. Stanev, Phys. Rev. D64:093010 (2001)

[6] D. Seckel \& T. Stanev, Phys. Rev. Lett., 95:141101 (2005)

[7] J. Abraham et al. (Auger collaboration), NIM A523. 50 (2003)

[8] P. Sokolsky (for the HiRes Collaboration), AIP Conf. Proc., 579, 296 (2001)

[9] H. Tokuno et al. (TA Collaboration), NIM A676, 54 (2012)

[10] V. Berezinsky, A.Z. Gazizov \& S.I. Grigorieva, Phys.Rev. D74:043005 (2006)

[11] J. Abraham et al, Phys.Rev.Lett. 101:061101 (2008)

[12] R.U. Abbasi et al, Phys.Rev.Lett. 100:101101 (2008)

[13] R. Aloisio, V. Berezinsky \& A. Gazizov, Astropart. Phys., 34, 620 (2011)

[14] A. Achterberg et al. (IceCube Collaboration), Astropart. Phys., 26, 155 (2006)

[15] M.G. Aartsen et al. (IceCube Collaboration), Phys. Rev. Lett. 111:021103 (2013)

[16] J. Abraham et al (Auger Collaboration), Phys. Rev. Lett. 100: 211101 (2008)

[17] I. Kravchenko et al (RICE Collaboration) Phys. Rev. D73: 082002 (2006)

[18] P. Gorham et al (Anita collaboration) Phys. Rev. D82: 022004 (2010); err. Phys. Rev. D85: 049901 (2012)

[19] A. Aab et al. (Auger Collaboration), arXiv:1307.4639, p.76 